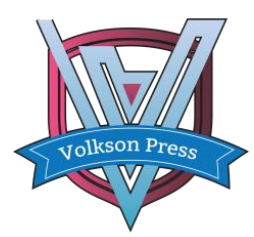

Contents List available at VOLKSON PRESS

Mechanical and Control Engineering (MCE)

DOI : http://doi.org/10.26480/wsmce.01.2017.01.05

\title{
LONGITUDINAL THERMAL STRESS/STRAIN ANALYSIS FOR CARBON FIBER COMPOSITE
}

\author{
Xiao Yao, Li Shulin*, Zhang Tiejun, Yin Junjie, Zhang Xianhang \\ Air Force Engineering University Xi'an, China \\ *Corresponding Author email: l.s.lin2008@163.com \\ This is an open access article distributed under the Creative Commons Attribution License, which permits unrestricted use, distribution, and reproduction in \\ any medium, provided the original work is properly cited
}

\section{ARTICLE DETAILS}

\section{Article History:}

Received 02 october 2017

Accepted 06 october 2017

Available online 11 november 2017

\section{Keywords:}

composite, thermal stress/strain, micro-mechanics, finite element model

\section{ABSTRACT}

Three different models were established to study thermal stress/strain of composite. Conventional lamellae model was simple and easy, but its accuracy was lower. Modified micro-mechanical model came from micromechanics. The temperature dependence of thermal expansion coefficient and orthotropic property of fiber were considered in this model, and the numerical result was agreed with finite element model. Composite thermal stress/strain under different temperature conditions $\left(40^{\circ} \mathrm{C}, 55^{\circ} \mathrm{C}, 70^{\circ} \mathrm{C}\right.$ ) were calculated by different models. The result indicated that thermal stress/strain of fiber and matrix increase almost linearly with increasing temperature. The calculation results of random and regularly spaced multi-fiber distribution model were shown that regularly spaced model could reduce stress range of fiber or matrix stress, reduce stress concentration, and avoid matrix cracking and interfacial deboning.

\section{Introduction}

Carbon/epoxy composite materials with low density, high strength, high modulus, thermal expansion coefficient, thermal conductivity, electromagnetic shielding and so on a series of excellent properties, are widely used in military and civil industrial fields, such as aircraft fuselage, wings and other structural components. The composite material is easily influenced by environmental factors during use or storage. When the ambient temperature changes, the fiber/matrix coefficient of thermal expansion mismatch and the difference of the ply angle will lead to thermal expansion stress. The thermal mismatch stress inside the structure weakens the fiber/Matrix interface, but also will promote the material to produce internal cracks, expansion and convergence, and ultimately lead to material failure, seriously affecting the service life of composite materials. Therefore, the study of the thermal stress of composite materials at different temperatures is beneficial to ensure the good bearing capacity and improve the service performance of composite materials.

The mechanical properties and thermal expansion coefficient of fiber and matrix are different in composite materials, and the thermal residual stress will be induced when the curing temperature drops to room temperature. Many domestic and foreign scholars have studied the residual thermal stress caused by the thermal expansion mismatch between the reinforcing phase and the matrix during the curing process of composites [1-6], theory and simulation [7-10]. Guemes [1] used fiber Bragg grating sensors to measure the longitudinal and transverse stresses of the composite laminates during the curing and cooling phases. $\mathrm{Wu}$ Zhanjun [6] used a single fiber Bragg grating in the curing process of fiberreinforced composites at different stages of monitoring the different key target parameters, including the molding phase of the resin temperature, the pressure applied when the resin pressure inside the curing and cooling stage laminar stress .Ding et al.[10] fully considered the influence of thermal expansion coefficient, chemical shrinkage, stress relaxation and other factors on thermal stress in the process of curing. The finite element simulation was carried out by calling ABAQUS subroutine, and compared with the traditional model. Chen Shuxian [11] established a mathematical model of temperature and thermal stress field. The internal temperature and thermal stress distribution of 3234/T300 laminates during curing were obtained by finite element analysis. Simulation shows that prolonging the pre-curing time and decreasing the heating rate can reduce the peak temperature of the center of the laminate and reduce the temperature gradient and thermal stress in the laminate.

Domestic research on the thermal stress caused by the change of ambient temperature in the process of using composite materials has been less studied [12,13], and the composite structure is the main part. The thermal expansion stress/strain on the mesoscopic surface directly affects the internal damage of the composite Evolution and macroscopic mechanical properties of the composites. Therefore, in this paper, the longitudinal thermal stress/strain of the composite material is taken as the research object, the traditional flaky model is summarized, the improved mesomechanics model is established, and the two models are verified by the finite element model. Finally, the effect of different fiber distributions on the longitudinal stress/strain of fibers and matrix is discussed.

\section{CLASSIC SHEET CALCULATION MODEL}

The classical sheet model assumes that the fiber and matrix are isotropic, and the resin and matrix expand freely without external force. The elongation of the two is different, but the internal stress is caused by bonding. The internal stress eliminates the elongation difference [14] caused by the different expansion of the fiber and matrix. According to the balance condition of force and strain coordination, the stress/strain of fiber and resin after expansion is calculated as follows: Longitudinal deformation coordination condition

$\varepsilon_{f 1}=\varepsilon_{m 1}$

$\varepsilon_{f 1}=\frac{\sigma_{f 1}}{E_{f 1}}+\alpha_{f 1} \Delta T, \varepsilon_{m 1}=\frac{\sigma_{m 1}}{E_{m}}+\alpha_{m} \Delta T$

Longitudinal force balance condition

$\sigma_{f 1} V_{f}+\sigma_{m 1} V_{m}=0$

Union of the above all kinds, get 
$\sigma_{f 1}=E_{f 1} E_{m} V_{m} \frac{\alpha_{m}-\alpha_{f 1}}{E_{f 1} V_{f}+E_{m} V_{m}} \Delta T$

$\sigma_{m 1}=E_{f 1} E_{m} V_{f} \frac{\alpha_{f 1}-\alpha_{m}}{E_{f 1} V_{f}+E_{m} V_{m}} \Delta T$

$\varepsilon_{f 1}=\varepsilon_{m 1}=\frac{\alpha_{f 1} E_{f 1} V_{f}+\alpha_{m} E_{m} V_{m}}{E_{f 1} V_{f}+E_{m} V_{m}} \Delta T$

$\alpha_{m}$ and ${ }^{\alpha_{f}}$ are the thermal expansion coefficient of resin and fiber; ${ }^{V_{m}}$ and $V_{f}$ are the volume fraction of resin and fiber; ${ }^{\sigma_{f}}$ and ${ }^{\sigma_{m}}$ are the internal stress caused by the thermal expansion of fiber and resin; ${ }^{\varepsilon_{f}}$ and $\varepsilon_{m}$ are the strain of fiber and resin; $\Delta T$ is the temperature increment.

The above derivation process does not involve the specific shape and arrangement of the fiber cross-section, so it has a relatively universal meaning. Many experiments and other accurate analysis methods show that this method is simple and easy to implement and has good practical value. The thermal stress and deformation in the direction perpendicular to the fiber are much more complex than in the longitudinal direction. This model is too simple, so errors in prediction of transverse mechanical properties are larger and the accuracy is much worse than in the longitudinal direction [15].

\section{IMPROVED MICROMECHANICS MODEL}

\subsection{Basic principle}

When the elastic constants of composite materials are solved by micromechanics, the series model or the parallel model and the material mechanics are often combined [15].The improved micromechanics model assumes that the fibers of unidirectional composites are square, taking $1 / 4$ of the square cell, and the fiber is simplified as a rectangular cross section, and the matrix is simplified as an equal thickness slice [16].In the actual transverse deformation of the composite, the fiber and the matrix both have the stress consistent (Series) part, and also have the strain consistent (parallel) part. If the strain is uniform and the matrix is $\lambda$ of the total matrix, the stress consistent part is $1-\lambda$, as shown in figure 1.The subscripts 11 and 22 in the figure indicate the longitudinal and transverse directions of the fiber, and the superscripts 1 and 2 respectively indicate the portions where the matrix and the fiber are transversely deformed and the strain and the stress are uniform.

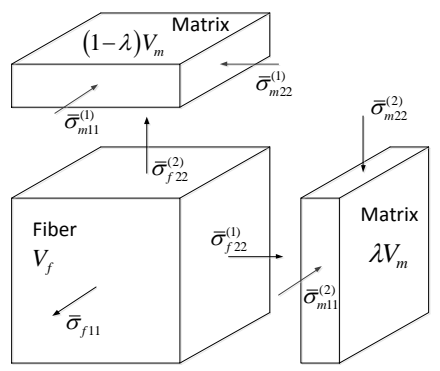

Figure 1: Modified micro-mechanical model

Stress balance equation along fiber direction

$V_{f} \bar{\sigma}_{f 11}=\lambda V_{m} \bar{\sigma}_{m 11}^{(2)}+(1-\lambda) V_{m} \bar{\sigma}_{m 11}^{(1)}$

Transverse stress balance equation

Stress consistent part:

$V_{f} \bar{\sigma}_{f 22}^{(1)}=(1-\lambda) V_{m} \bar{\sigma}_{m 22}^{(1)}$

Strain consistent part:

$V_{f} \bar{\sigma}_{f 22}^{(2)}=\lambda V_{m} \bar{\sigma}_{m 22}^{(2)}$

Longitudinal deformation conditions Stress consistent part:

$$
\begin{aligned}
& \alpha_{f 11} \Delta T+\frac{\bar{\sigma}_{f 11}}{E_{f 11}}-\frac{v_{f 12} \bar{\sigma}_{f 22}^{(1)}}{E_{f 11}}-\frac{v_{f 12} \bar{\sigma}_{f 22}^{(2)}}{E_{f 11}}= \\
& \alpha_{m} \Delta T-\frac{\bar{\sigma}_{m 11}^{(1)}}{E_{m 11}}+\frac{v_{m} \bar{\sigma}_{m 22}^{(1)}}{E_{m 22}}
\end{aligned}
$$

Strain consistent part:

$$
\begin{aligned}
& \alpha_{f 11} \Delta T+\frac{\bar{\sigma}_{f 11}}{E_{f 11}}-\frac{v_{f 12} \bar{\sigma}_{f 22}^{(1)}}{E_{f 11}}-\frac{v_{f 12} \bar{\sigma}_{f 22}^{(2)}}{E_{f 11}}= \\
& \alpha_{m} \Delta T-\frac{\bar{\sigma}_{m 11}^{(2)}}{E_{m 11}}+\frac{v_{m} \bar{\sigma}_{m 22}^{(2)}}{E_{m 22}}
\end{aligned}
$$

Lateral deformation condition Stress consistent part:

$$
\begin{aligned}
& \alpha_{f 22} \Delta T-\frac{v_{f 12} \bar{\sigma}_{f 11}}{E_{f 11}}+\frac{\bar{\sigma}_{f 22}^{(1)}}{E_{f 22}}= \\
& \alpha_{m} \Delta T+\frac{v_{m} \bar{\sigma}_{m 11}^{(1)}}{E_{m 11}}-\frac{\bar{\sigma}_{m 22}^{(1)}}{E_{m 22}}
\end{aligned}
$$

Strain consistent part:

$$
\begin{aligned}
& \alpha_{f 22} \Delta T-\frac{v_{f 12} \bar{\sigma}_{f 11}}{E_{f 11}}+\frac{\bar{\sigma}_{f 22}^{(2)}}{E_{f 22}}= \\
& \alpha_{m} \Delta T+\frac{v_{m} \bar{\sigma}_{m 11}^{(2)}}{E_{m 11}}-\frac{\bar{\sigma}_{m 22}^{(2)}}{E_{m 22}}
\end{aligned}
$$

Assuming that the carbon fibers are orthorhombic, then

$\frac{v_{f 12}}{E_{f 11}}=\frac{v_{f 21}}{E_{f 22}}$

The resin is isotropic, then

$$
E_{m 11}=E_{m 22}=E_{m}
$$

The relationship between the coefficient of thermal expansion and the temperature of the resin is [17]

$\alpha_{m}=\alpha_{m(R T)}\left[\frac{T_{g}-T}{T_{g}-T_{R T}}\right]^{-1 / 2}$

The longitudinal strain solution equation is

$$
\varepsilon_{L}=\alpha_{m} \Delta T-\frac{\bar{\sigma}_{m 11}^{(1)}}{E_{m 11}}+\frac{v_{m} \bar{\sigma}_{m 22}^{(1)}}{E_{m 22}}
$$

\subsection{Examples and results}

Taking carbon fiber / epoxy matrix composites as an example, the longitudinal and transverse stresses/strains produced by thermal expansion of fiber and matrix during $T=70^{\circ} \mathrm{C}$ (i.e. $\Delta T=50^{\circ} \mathrm{C}$ ) are calculated. The mechanical properties and physical parameters of fibers and resins are shown in table 1 .

The parameters of Table 1 are substituted into the above-mentioned ones, and the ratio of the matrix and the transverse strain of the fiber in the reference [18] is taken as $\lambda=0.435$. The stress of the fiber and the matrix in different directions of heating up 50 degrees is listed in Table 2 , and the longitudinal strain $\varepsilon_{L}=133.92 \mu \varepsilon$ is calculated.

Table 1: Properties of carbon fiber composite 


\begin{tabular}{|c|c|c|c|c|c|c|c|}
\hline$V_{f}$ & $V_{m}$ & $E_{f 11} / G P a$ & $E_{f 22} / G P a$ & $v_{f 12}$ & $\alpha_{f 11}{ }^{\circ} \mathrm{C}$ & $\alpha_{f 22} I^{\circ} \mathrm{C}$ & $E_{m} / G P a$ \\
\hline 0.3 & 0.7 & 252 & 25.2 & 0.28 & $0.12^{*} 10^{-6}$ & $2.7^{*} 10^{-6}$ & 3.5 \\
\hline$v_{m}$ & $\alpha_{m(\{T T)} / C^{-1}$ & $T_{R T} /{ }^{\circ} \mathrm{C}$ & $T_{g} /{ }^{\circ} \mathrm{C}$ & & & & \\
\hline 0.35 & $62.7^{*} 10^{-6}$ & 20 & 186 & & & & \\
\hline
\end{tabular}

Table 2: Longitudinal and transverse thermal stress of fiber and matrix

\begin{tabular}{ccccccc}
\hline$\sigma_{f 11}$ & $\sigma_{f 22}^{(1)}$ & $\sigma_{f 22}^{(2)}$ & $\sigma_{m 11}^{(1)}$ & $\sigma_{m 22}^{(1)}$ & $\sigma_{m 11}^{(2)}$ & $\sigma_{m 22}^{(2)}$ \\
\hline 43 & 19.35 & 19.35 & 18.46 & 16.59 & 18.46 & 16.59 \\
\hline 4. & \multicolumn{7}{l}{ FINITE ELEMENT SIMULATION MODEL }
\end{tabular}

\subsection{Single fiber thermal stress/strain simulation model}

In order to verify the two models of thermal stress/strain theory, a finite element model of fiber and matrix is established, as shown in figure 2 . The total number of units in the model is 124000 , and the fiber volume fraction is $30 \%$. The symmetric boundary conditions and the predefined field [19] are applied.

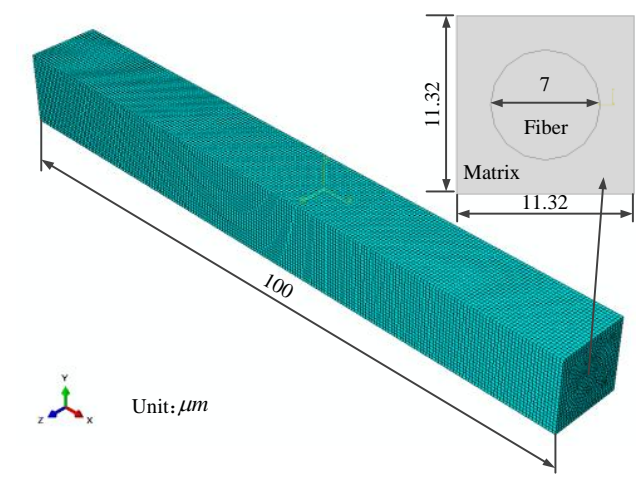

Figure 2: Single fiber finite element model

Enter the material and physical parameters of the fiber and matrix in Table 1 , and calculate the longitudinal stress/strain cloud as shown in Figure 3 and Figure 4. The free-end stress-strain cloud of the model is more complicated because of the free end of the cloud. To eliminate the difference between the elongation of the fiber and the resin, complicated internal stresses are required. The distribution of stress/strain in the inner region of the model is more uniform. From the cross-section cloud in the middle part of the model, it can be seen that the longitudinal strain of the fiber and the matrix is about $130 \mu \varepsilon$, the fiber longitudinal stress distribution is relatively uniform, the thermal stress is $35 M P a$ and the longitudinal thermal stress is $16 M P a$.

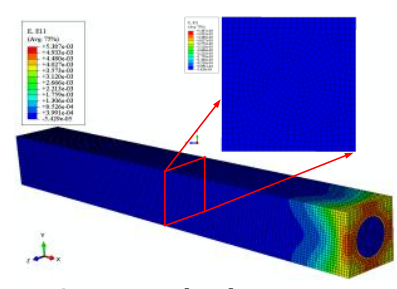

Figure 3: Longitudinal strain Contour

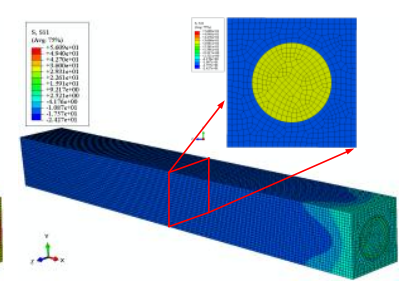

Figure 4: Longitudinal stress contour

\subsection{Uniform distribution of multi-fiber thermal stress/strain simulation model}

The multi-fiber thermal expansion stress/strain was studied by taking the fiber volume fraction $30 \%$ (see Figure 5) and the number of fibers 20 as an example. Simulation results shown in Figure 6,7. According to 6,7, the stress/strain distribution of the fiber uniform distribution model is similar to that of the single fiber, that is, the longitudinal strain is consistent, and the stress distribution of the fiber and the matrix is uniform.

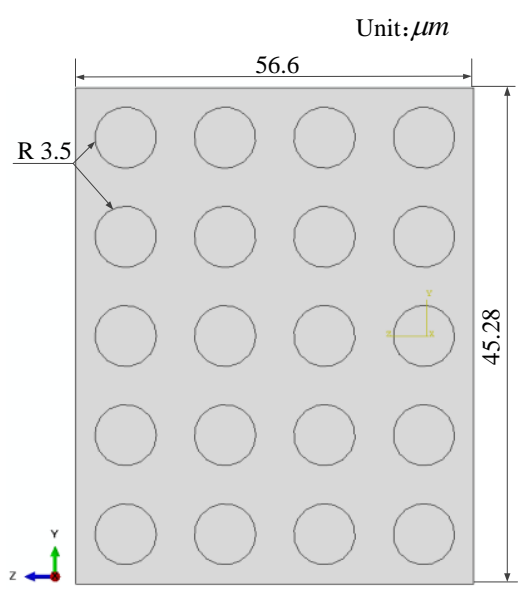

Figure 5: Regularly spaced multi-fiber distribution model

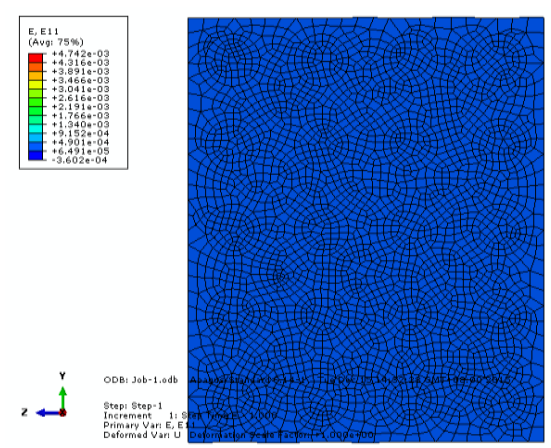

Figure 6: Longitudinal strain contour of regularly spaced multi-fiber distribution model

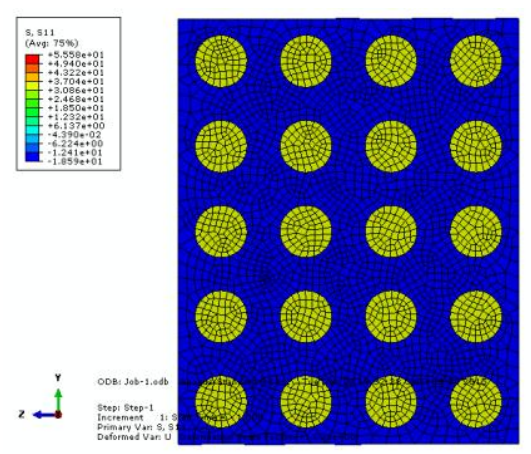

Figure 7: Longitudinal stress contour of regularly spaced multi-fiber distribution model

4.3 Random distribution of multi-fiber thermal stress / strain simulation model

The fiber volume fraction and number of the fiber random distribution model are consistent with the uniform distribution model, but the fiber spatial distribution is random irregular distribution, and the longitudinal stress/strain calculation results are shown in Figs. 8 and 9.From the longitudinal strain cloud, it can be seen that the longitudinal strain of the fiber is basically the same as that of the substrate, while the longitudinal stress distribution of fiber and resin is no longer uniform, the local stress of a number of fibers is obviously higher than that of other fibers, and the stress concentration phenomenon appears in the matrix of fiber dense area. 


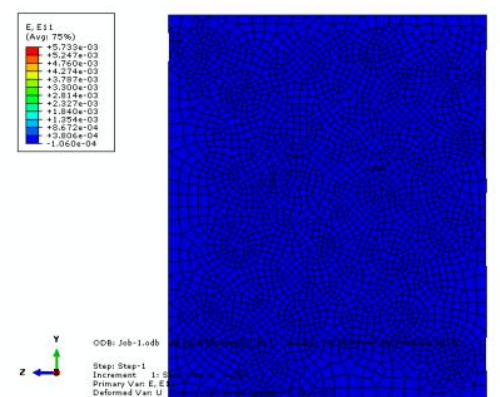

Figure 8: Longitudinal strain contour of random spaced multi-fiber distribution model

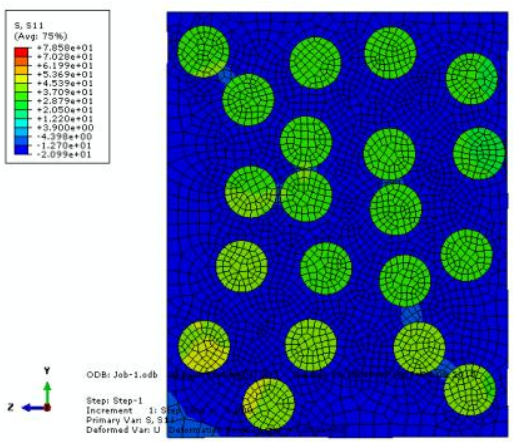

Figure 9: Longitudinal stress contour of random spaced multi-fiber distribution model

\section{RESULTS ANALYSIS AND DISCUSSION}

5.1 Comparison of three thermal stress/strain calculation models at different temperatures

When the ambient temperature is $40^{\circ} \mathrm{C}, 55^{\circ} \mathrm{C}$ and $70^{\circ} \mathrm{C}$ (reference temperature is $20^{\circ} \mathrm{C}$ ), the results of three models of thermal expansion of fiber and matrix are compared with those shown in figure 10. It can be found that the sheet model and the modified micromechanical model agree well with the finite element results, but the improved micromechanical model has smaller error and higher accuracy. In addition, with the increase of temperature, the longitudinal stress/strain of fiber and matrix increase gradually, and approximately obey the linear relationship. The stress and strain of the fiber or resin calculated by the improved micromechanical model are slightly higher than that of the finite element model, which shows that the model is conservative and suitable for predicting damage and damage safely.

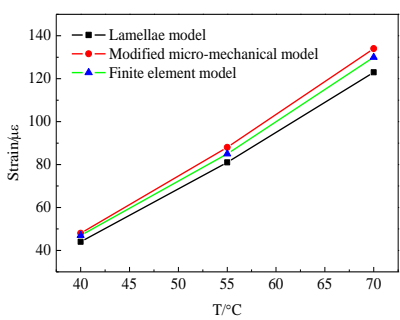

(a) Longitudinal strain

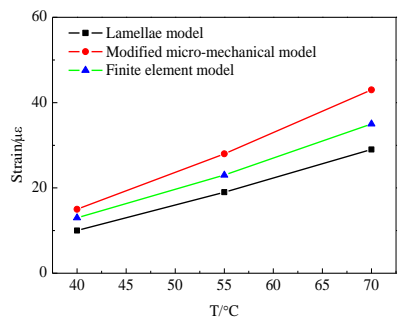

(b) Longitudinal stress of fiber

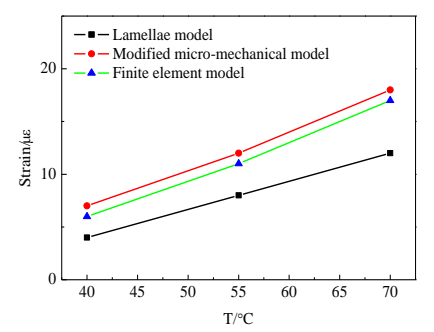

(c) Longitudinal stress of matrix

An important parameter in the improved micromechanical model is that the strain agrees with the matrix and accounts for A of the total matrix. When the A changes, the longitudinal and transverse thermal stress/strain curves of the fiber / matrix are shown in figure 11.It can be seen from Fig.11 that the longitudinal strain and fiber longitudinal stress have little change, the strain is about $133 \mu \varepsilon$, and the stress is about $43 M P a$, because the longitudinal stress balance equation and the deformation coordination condition are determined by the content of each component of the composite material while the fiber volume fraction is constant $(30 \%)$, so the two have little change, which is consistent with the principle of flaky model. However, the lateral stress of fiber and matrix is greatly influenced by $\lambda$. With the increase of the uniform strain ratio, the stress of the consistent part of the matrix increases. As the proportion increases, the part of the matrix will bear more and more loads Increase; fiber transverse stress showed obvious inverse proportion characteristics, at 0.5 , both equal, because the model has a symmetrical character.

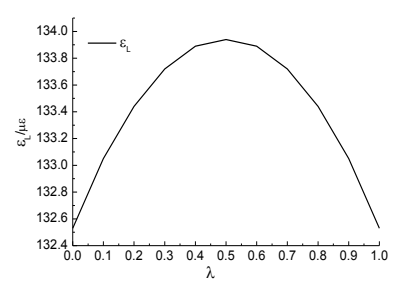

(a)Longitudinal strain

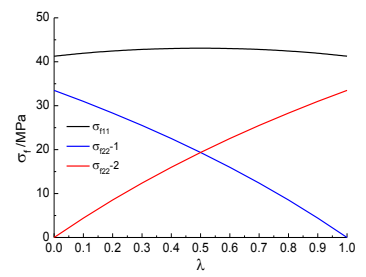

(b) Longitudinal and transverse stress of fiber

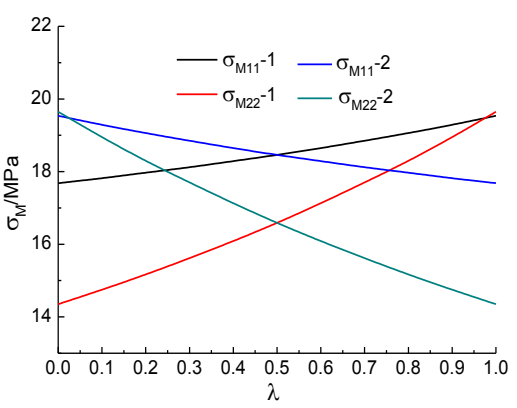

(c) Longitudinal and transverse stress of matrix

Figure 11: Changes of stress/strain with $\lambda$

\subsection{Single fiber transverse thermal stress/strain analysis}

The transverse thermal stress/strain nephogram of a single fiber is shown in figure 12. Single fiber transverse thermal stress / strain cloud shown in Figure 12. From the finite element simulation results, it can be seen that the distribution of the stress S22 at the top and the right of the substrate is consistent, that is, the stress of the substrate near the interface is larger and the stress of the external substrate is smaller. The strain E22 at the top increases gradually from the inside to the outside, while the strain at the right end decreases gradually. This is due to the symmetry of the resin at the right end along the $\mathrm{Z}$ axis and the free expansion of the $\mathrm{Y}$ direction. The strain has a certain counteracting effect, and the closer to the central line, the more obvious the offset is, i.e., the minimum strain.

The stress and strain distributions at the top and right ends of the fiber are relatively uniform, basically in the order of magnitude $(7.5 \mathrm{MPa} / 300 \mu \varepsilon$ ), with little fluctuation. From the stress cloud image, the stress value at the top of the resin is consistent with the stress value in the fiber direction, while the sign at the right end is opposite, that is, the top is consistent with the stress of the fiber. Strain moiré patterns can be observed with the color of the right moiré pattern (light blue) The right-hand moire patterns are similar in color (blue) and the tops differ greatly in color (red and blue), ie, the right-hand end corresponds to the fiber strain. The above conclusions verify the rationality and effectiveness of improving the mesomechanics model.

Figure 10: Comparison of three models under different temperature conditions 


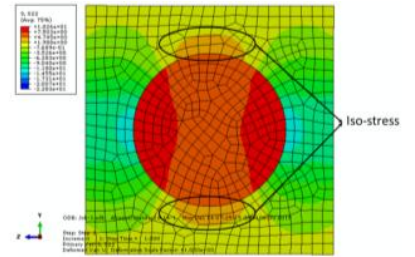

(a) Transverse stress

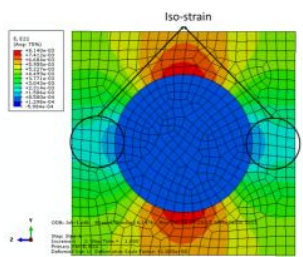

(b) Transverse strain
Figure 12: Transverse stress/strain contour of single fiber

\subsection{Effect of fiber distribution on longitudinal stress/strain}

The stress/strain results of the multi-fiber uniform distribution model are similar to those of the single fiber, that is, the longitudinal strain is consistent, and the stress distribution of the fiber and the matrix is uniform. However, the random distribution of the fibers makes the longitudinal stress distribution of the fiber and resin no longer uniform, the obvious stress concentration phenomenon, such as the number of root fibers local stress was significantly higher than other fibers, random model fiber longitudinal stress range ( $22-46 M P a$ ) wider than the uniform model ( $34.9-36.3 \mathrm{MPa}$ ). When the fiber is randomly distributed, the fiber is dense and the matrix is rich and poor, and the fibers with small spacing produce high stress, resulting in matrix cracking or interface debonding, which leads to strength damage and failure of composites $[20,21]$. Therefore, improving the uniformity of fiber distribution during the manufacturing of composites can effectively reduce thermal stress.

\section{CONCLUSION}

In this paper, the thermal expansions of composites with varying ambient temperature are investigated. The thermal expansion stress/strain of composites at different temperatures are calculated by using three models. The traditional flaky model is introduced. An improved mesomechanics model is established and validated by the finite element model. Through the comparison between different models, we can get the following conclusions:

(1) Both the sheet model and improved micromechanics model can predict the composite good longitudinal stress/strain well, the chip model is simple and easy to use as the classical theory, but the error is slightly larger. With the increase of temperature, fiber and matrix of longitudinal stress/strain increases gradually, which approximately obey the linear relationship. The comparison of the three models shows that the calculation results of the improved meso mechanical model are close to those of the finite element method.

(2) The single fiber transverse thermal stress/strain finite element results demonstrate the correctness of the improved micromechanics model, that is, some of the matrix stress / strain is consistent with the fiber strain or stress. The model derived from micromechanics, considering the relationship between thermal expansion coefficient and temperature, and fiber orthotropic properties, can effectively predict the thermal stress and strain of composites, such as $T=70^{\circ} \mathrm{C}$, the longitudinal strain $\varepsilon_{L}=133.92 \mu \varepsilon$, close to the finite element results( ${ }^{130 \mu \varepsilon}$ ).

(3) The uniform arrangement of fibers makes the distribution of thermal stress/strain more uniform, and the longitudinal thermal stress range ( $34.9-36.3 \mathrm{MPa}$ ) is obviously less than that of stochastic distribution model ( $22-46 M P a$ ). It can effectively reduce the concentration of thermal stress and prevent failure such as matrix cracking or interface debonding.

\section{REFERENCES}

[1] Guemes, J.A., Menéndez, J.M. 2002. Response of Bragg grating fiber-optic sensors when embedded in composite laminates [J]. Composite Science and Technology, 62 (7-8), 959-966.

[2] Sorensen, L., Gmür, T., Botsis, J. 2006. Residual strain development in an AS4/PPS thermoplastic composite measured using fibre Bragg grating sensors [J]. Composites Part A: Applied Science and Manufacturing, 37 (2), 270-281.
[3] Karalekas, D., Cugnoni, J., Botsis, J. 2008. Monitoring of process induced strains in a single fibre composite using FBG sensor: A methodological study [J]. Composites Part A: Applied Science and Manufacturing, 39 (7), 1118-1127.

[4] Khoun, L., deOliveira, R., Michaud, V., Hubert, P. 2011. Investigation of process-induced strains development by fibre Bragg grating sensors in resin transfer moulded composites [J]. Composites Part A: Applied Science and Manufacturing, 42 (3), 274-282.

[5] Canal, L.P., Benavente, M., Hausmann, M., Michaud, V. 2015. Process-induced strains in RTM processing of polyurethane/carbon composites [J]. Composites Part A: Applied Science and Manufacturing, 78 (4), 264-273.

[6] Zhanjun, W., Boming, Z., Libing, W. 2004. Multi-parameters monitoring for fiber reinforced plastics with a single fiber bragg gratings [J]. Acta Materiae Compositae Sinica, 21 (6), 82-86.

[7] Choo, H., Bourke, M.A.M., Daymond, M. R. 2001. A finite-element analysis of the inelastic relaxation of thermal residual stress in continuous-fiber-reinforced composites [J]. Composite Science and Technology, 61 (12), 1757-1772.

[8] Tsai, J.L., Chi, Y.K. 2008. Investigating thermal residual stress effect on mechanical behaviors of fiber composites with different fiber arrays [J]. Composites Part B: Engineering, 39 (4), 714-721.

[9] Pan, Y., Pelegri, A.A. 2008. Influence of matrix plasticity and residual thermal stress on interfacial debonding of a single fiber composite [J]. Journal of Mechanics of Materials and Structures, 5 (1), 128-142.

[10] Ding, A.X., Li, S.X., Sun, J.X., Wanga, J., Zua, L. 2016. A thermoviscoelastic model of process-induced residual stresses in composite structures with considering thermal dependence [J]. Composite Structures, 136, 34-43.

[11] Chen, S.X., Tian, H., Qin, W.F. 2014. 3D unsteady analysis of temperature and thermal stress fields of resin matrix composite during curing process [J]. Journal of University of Electronic Science and Technology of China, 43 (4), 547-551.

[12] Xi, Z.C., Chen, H.R. 1992. Analysis of thermal deformations and stresses in stiffened composite laminates [J]. Acta Materiae Compositae Sinica, 9 (4), 17-22.

[13] Feng, Y.Z., He, L.W. 2005. Thermal stress analysis for laminated composite plates [J]. Journal of Lanzhou University, 41 (6), 94-99.

[14] Shen, G.L., Hu, G.K., Liu, B. 2013. Mechanics of Composite Materials [M]. Beijing: Tsinghua University Press, 233-234.

[15] Wang, Z.M. 1990. Mechanics and structural mechanics of Composite Materials [M]. Beijing: China Machine Press, 134-138.

[16] He, Q.Q., Zhou, C.W., Zhou, C. 2014. Micro-mechanical model of thermal expansion/contraction for damaged fiber reinforced composites [J]. Acta Materiae Compositae Sinica, 31 (4), 1077-1083.

[17] China Aviation Academy. 2001. Composite structures design manual [M]. Beijing: Aviation Industry Press, 482-484.

[18] Li, L., Zhou, C.W., Binod, A. 2011. Micro-mechanical analysis of thermal expansion of damaged composites. Journal of Engineering and Applied Sciences, 6 (6), 408-415.

\section{[19] Abaqus Analysis User's Manual [M]. (2009). Version6.9;}

[20] Lu, C., Chen, P., Yu, Q., Gao, J., Yu, B. 2008. Thermal residual stress distribution in carbon fiber/novel thermal plastic composite [J]. Applied Composite Materials, 15 (3), 1077-1083.

[21] Huang, Y., He, F., Wang, Y.Z. 2009. Testing and characterization of hygrothermal stresses in carbon-fibers reinforced epoxy composites using Raman spectroscopy [J]. Acta Materiae Compositae Sinica, 26 (4), 22-28. 